Renormalisation group determination of the order of the DNA denaturation transition

This content has been downloaded from IOPscience. Please scroll down to see the full text. 2010 EPL 8940011

(http://iopscience.iop.org/0295-5075/89/4/40011)

View the table of contents for this issue, or go to the journal homepage for more

Download details:

IP Address: 150.214.182.116

This content was downloaded on 30/03/2016 at 10:01

Please note that terms and conditions apply. 


\title{
Renormalisation group determination of the order of the DNA denaturation transition
}

\author{
J. M. Romero-Enrique ${ }^{1}$, F. De los $\operatorname{Santos}^{2,3}$ and M. A. Muñoz ${ }^{2,3}$ \\ ${ }^{1}$ Departamento de Física Atómica, Molecular y Nuclear, Universidad de Sevilla - Apartado de correos 1065, \\ 41080 Seville, Spain, EU \\ ${ }^{2}$ Departamento de Electromagnetismo y Física de la Materia, Universidad de Granada - Fuentenueva s/n, \\ 18071 Granada, Spain, EU \\ ${ }^{3}$ Instituto Carlos I de Física Teórica y Computacional, Universidad de Granada - Fuentenueva s/n, \\ 18071 Granada, Spain, EU
}

received 3 November 2009; accepted in final form 12 February 2010

published online 9 March 2010

PACS 02.50.-r - Probability theory, stochastic processes, and statistics

PACS 64.60.F- - Equilibrium properties near critical points, critical exponents

PACS 87.14.G- - Nucleic acids

\begin{abstract}
We report on the nature of the thermal-denaturation transition of homogeneous DNA as determined from a renormalisation group analysis of the Peyrard-Bishop-Dauxois model. Our approach is based on an analogy with the phenomenon of critical wetting that goes further than previous qualitative comparisons, and shows that the transition is continuous for the average basepair separation. However, since the range of universal critical behaviour appears to be very narrow, numerically observed denaturation transitions may look first-order, as it has been reported in the literature.
\end{abstract}

Copyright (c) EPLA, 2010

Introduction. - In addition to its central relevance in biology, the DNA molecule also displays a variety of remarkable physical properties, some of which are key to the understanding of DNA function [1]. For instance, mechanical properties such as bending, twisting, or compression are directly related to DNA replication or transcription, which requires that the two strands are separated in order that they can be read by DNA or RNA polymerase. This can be achieved by various mechanisms, including pulling enzymes, mechanical force or gentle heating. In this latter case, the process is known as DNA thermal denaturation or DNA melting, and has received a great deal of attention over several decades [2]. Experimental observation of the fraction of bound pairs or the average base pair separation as a function of temperature reveals a sharp jump in the denaturation curves from double- to single-stranded DNA, a behaviour that hints at some sort of phase transition. However, controversies remain regarding the nature of this transition (whether first- or second-order), see below. This point goes beyond mere physical curiosity and has biological relevance, for there is increasing interest in the correspondence between functional and thermodynamic melting properties, e.g., the identification of coding sequences in genomes on the basis of thermodynamic melting behaviour [3].
Models of varying complexity and applicability have been developed that account for DNA melting. Two large families are based on the models of Poland-Scheraga (PS) [4] and Peyrard-Bishop-Dauxois (PBD) [5], or modifications thereof. Within the PS framework, the DNA is described as a sequence of base pairs that can be either bound or unbound. Thermal fluctuations cause segments of DNA to unbind, creating temporarily denaturated loops of variable size which can ultimately coalesce upon increasing the temperature, thus triggering the denaturation transition. It has been shown that the entropy contribution of the loops depends on their size, $l$, as $\sim 1 / l^{c}$, and three different scenarios have been reported depending only on the value of $c: c \leqslant 1$, no phase transition, $1<c \leqslant 2$, continuous transition, and $c>2$, first-order phase transition. The value of $c$ is not easily determined, but it has been recently demonstrated that taking into account the excluded-volume interactions between denaturated loops and the rest of the chain is enough to give $c>2[6]$, so the transition is therefore first-order (see [7] for a refined analysis of these issues and a discussion of some open questions).

Turning to PBD-type models, the situation is much less clear. The PBD model considers only the stretching between corresponding base pairs (see details in the next 
section). The transition proceeds as described for the PS, but including intermediate states because the stretching is a continuously varying variable. It has been thoroughly studied by means of Monte Carlo simulations, Langevin dynamics, path integral methods and different transfer integral approaches, but the question of the order of the transition remains as yet unsettled. Claims have been reported in the literature that the transition is first-order yet with a diverging correlation length, asymptotically second-order although very sharp looking in appearance, while other studies are inconclusive. We shall return to this point in the next section.

In this letter we take on the question of the order of the DNA denaturation transition in the PBD model by means of an exact renormalisation group analysis. Our approach is based on an analogy with the phenomenon of critical wetting that goes further than previous qualitative comparisons. In the next section, we review the $\mathrm{PB}$ and PBD models. Next, we explain the analogy with wetting and then proceed with the renormalisation group calculation, which shows that the transition is continuous for the average base pair separation. A summary with our conclusions is presented in the final section.

The Peyrard-Bishop-Dauxois model of DNA. The Peyrard-Bishop model ignores the helicoidal structure of the DNA molecule and the properties associated with it, and focuses on the stretching of the hydrogen bonds connecting base pairs, which are represented by continuous variables $h_{n}(n=1,2, \ldots, N$, where $N$ is the length of the chain). For homogeneous samples (only AT of GC pairs), the Hamiltonian of the model reads [5]

$$
H=\sum_{n=1}^{N}\left[\frac{p_{n}^{2}}{2 m}+W\left(h_{n}, h_{n-1}\right)+V\left(h_{n}\right)\right],
$$

where the first term is the kinetic energy for bases of mass $m, W\left(h_{n}, h_{n-1}\right)=k\left(h_{n}-h_{n-1}\right)^{2}$ describes the harmonic stacking interaction between neighbouring bases, and $V$ represents the average potential between the two bases in a pair which is modeled by a Morse potential, $V\left(h_{n}\right)=$ $D\left(e^{-a h_{n}}-1\right)^{2}$. Note that the asymmetry of the two strands is neglected in that a common mass $m$ for the bases is used and the same stacking coefficient $k$ along the chain is assumed. $D$ is the dissociation energy of the pairs and $a$ denotes the spatial range of the potential. Their precise values, which are unimportant for our purposes, can be determined from the fitting of DNA experimental denaturation curves [8]. Two standard observables are the average stretching $\langle h\rangle$ and the density of bound base pairs $\left\langle e^{-h}\right\rangle$.

It was soon recognised that the simple PB model needed to be improved if it was to properly account for the sharp shape of the experimental denaturation curves. This was achieved by changing from a harmonic stacking interaction to the nonharmonic one

$$
k \rightarrow k\left[1+\rho e^{-\alpha\left(h_{n}+h_{n-1}\right)}\right]
$$

whose origin lies in the change in the electronic distribution on the bases when the hydrogen bonds are broken and that provides a more realistic treatment of the phosphate backbone stiffness. This new term leads to very sharp melting transitions at substantially reduced denaturation temperatures [9].

Let us now briefly review the different scenarios that have been reported for the denaturation transition depending on the stiffness parameter $\rho$, for both homogeneous and heterogeneous DNA samples. There is a consensus that, in the simplest case $\rho=0$, second-order denaturation transitions are observed irrespective of the composition of the sample (whether heterogeneous or homogeneous) [10,11]. A nonvanishing $\rho$ and heterogeneous sequences successfully exhibit the characteristic abrupt, multistep melting observed in heterogeneous DNA molecules [10] for intermediate-length sequences (see also [12]). On the contrary, in the case of nonzero $\rho$ and homogeneous DNA the situation is much less clear. On the one hand, there is a claim by Cule et al. that the transition region is extremely narrow, making it very sharp in appearance although, asymptotically, it is expected to be second-order [10]. On the other hand, Dauxois et al. [9] reported a first-order transition, yet with a diverging correlation length [11]. Subsequent improved transfer-integral investigations by Joyeux et al. [13] did not settle the question, inasmuch as the numerics seems to indicate a first-order transition, but without discarding the possibility of a narrow second-order one. Finally, results from a very recent path integral investigation suggest that the denaturation of homogeneous DNA has the features of a second-order phase transition [14]. In what follows, we clarify this situation by exploiting an analogy with the phenomenon of wetting.

The wetting analogy. - The canonical partition function of the model factorises as usual into a product of kinetic and configurational parts, $Z=Z_{p} Z_{y}$, with $Z_{p}=$ $\left(2 \pi m k_{B} T\right)^{N / 2}$ and

$$
Z_{y}=\int \prod_{n=0}^{N} \mathrm{~d} h_{n} e^{-\beta H^{\prime}}
$$

where we have defined $H^{\prime}=\sum_{n}(W+V)$ as the configurational part of $H$. Given that most experiments on DNA thermal denaturation are performed in water, the kinetic term does not play any role, and hence we can restrict ourselves to the configurational part. In the continuum limit, for small values of $h_{n}-h_{n-1}$, the configurational part of the PBD Hamiltonian can be expressed as

$$
H_{e w}=\int \mathrm{d} x\left[\frac{k}{2}\left(1+\rho e^{-2 \alpha h}\right)(\nabla h)^{2}+w_{1} e^{-a h}+w_{2} e^{-2 a h}\right],
$$

where $w_{1}, w_{2}$ and $k$ are generic parameters. For $\rho=0 H_{e w}$ is the standard interfacial Hamiltonian for equilibrium critical wetting transitions in the presence of short-ranged forces, that is, the unbinding of the interface separating 
two coexisting phases from a substrate, which occurs upon increasing the temperature [15]. It constitutes an approximation to the PBD Hamiltonian that disregards the kinetic terms, but from which equilibrium information can be gleaned. The two strands of the DNA molecule correspond to the substrate and the interface in the wetting context, and the denaturation of the former to the unbinding of the latter. The analogy also extends to heterogeneous sequences, the thermal denaturation of heterogeneous DNA corresponding to the wetting of a one-dimensional interface from a disordered substrate. This formal relation between wetting and DNA thermal denaturation was already noticed by Fisher [16] and exploited by Cule and Hwa [10] and by Ares et al. [17]. The analogy was carried further in [18], where it was pointed out that the reported critical exponents characterising the DNA denaturation transition in the homogeneous case, $\langle h\rangle \sim|\delta|^{\beta}$ and $\xi \sim|\delta|^{-\nu}$ (where $\delta=\left(T-T_{c}\right) / T_{c}$ and $\xi$ is the correlation length) [11], are those of two-dimensional critical wetting, $\beta=-1$ and $\nu=2[15]$. It was also shown by numerical simulations that the average stretching $\langle h\rangle$ diverges as $t^{1 / 4}$ at the transition temperature, in agreement with the exact result for the thickness of the wetting layer [19]. Furthermore, the density of closed base pairs [10] scales as the surface order-parameter in wetting, $\left\langle h^{-1}\right\rangle \sim|\delta|[15]$.

Interestingly, it was also argued in [18] that the theory of critical wetting should also apply to the PBD model (nonzero- $\rho$ ). Renormalisation group analyses of threedimensional critical wetting as embodied in eq. (4) with $\rho=0$ famously predict a strong non-universal critical behaviour [20]. These predictions, however, are at odds with extensive Ising model computer simulations due to Binder et al. [21] as well as with experiments [22], which yield a mean-field-like second-order phase transition for the wetting problem. Fisher and Jin [23] suggested that this discrepancy arises from fundamental defects in the wetting Hamiltonian

$$
H_{e w}(\rho=0)=\int \mathrm{d} x\left[k(\nabla h)^{2}+w_{1} e^{-a h}+w_{2} e^{-2 a h}\right],
$$

which should include a variable, position-dependent interfacial stiffness

$$
k(h)=k+w_{1}^{\prime} e^{-a h}+w_{2}^{\prime} a h e^{-2 a h}+\cdots .
$$

When supplemented with the corrected stiffness, the structure of the wetting Hamiltonian eq. (5) is very similar to that of the PBD one, eq. $(4)^{1}$. A more detailed comparison reveals that in critical wetting the parameter $w_{1}^{\prime}$

\footnotetext{
${ }^{1}$ Just to complete the wetting story, the Fisher-Jin improved Hamiltonian did not yield the desired result, namely a crossover to mean-field-like behaviour. According to a linear renormalisationgroup study, the presence of the term proportional to $w_{2}^{\prime}>0$ is capable of destabilising the critical-wetting transition, driving the transition weakly first-order depending on system parameters in $d=3$ [23]. A subsequent investigation allowed the analysis to be extended concluding that a first-order transition can appear only for dimensions $d \geqslant 2.41$ [24]. This puzzling situation was clarified
}

vanishes linearly with the transition temperature and it is the next-to-leading term, $w_{2}^{\prime} e^{-2 a h}$, that controls the critical behaviour. On the contrary, for the PBD Hamiltonian $w_{1}^{\prime}=k \rho / 2>0$ is finite and $w_{2}^{\prime}$ is identically zero. Thus, a renormalisation group analysis of the PBD model along the same lines as in the wetting case requires switching on a nonvanishing $w_{1}^{\prime}$ and truncating the series to firstorder. Despite these differences, we shall show that one can avail from standard renormalisation group techniques developed for the wetting problem to draw conclusions on the order of the DNA denaturation transition. Such an analysis is carried out in the next section, where we prove that the one-dimensional melting transition for homogeneous DNA sequences is continuous in $\langle h\rangle$.

Exact decimation procedure. - The wetting analogy allows us to perform an exact decimation renormalisation-group (RG) [26] analysis of the DNA denaturation in the PBD model. This RG procedure was successfully applied to the 2D wetting transition [27] $\left(H_{e w}(\rho=0)\right)$ where the non-trivial fixed points can be analytically calculated for short-ranged forces [28], and also for more general interactions [29]. These calculations can be straightforwardly extrapolated to the PBD model with $\rho \neq 0$ which, as shown before, is formally equivalent to the discrete version of the standard interfacial Hamiltonian for the 2D wetting transition because the position-dependent stacking interaction in the PBD model is the analogous of the position-dependent stiffness in the wetting case. Recent studies show that the dependence on the position of the effective stiffness can induce new critical phenomena, and it can even drive the transition first-order [30].

The configurational part of the PBD Hamiltonian can be written as $H^{\prime}=\sum_{i=1}^{N} H_{i}^{(0)}\left(h_{i}, h_{i+1}\right)$, where $H_{i}^{(0)}=W\left(h_{i}, h_{i+1}\right)+\left(V\left(h_{i}\right)+V\left(h_{i+1}\right)\right) / 2$. By simplicity we shall consider periodic boundary conditions $h_{1}=h_{N+1}$, although this choice will not affect our conclusions. We define the initial transfer matrix as $T^{(0)}\left(h_{i}, h_{i+1}\right)=\exp \left(-\beta H_{i}^{(0)}\right)$. Note that $T^{(0)}$ is symmetrical under an exchange of its arguments. The decimation RG procedure defines the transfer matrix $T^{(n)}$ at any $\mathrm{RG}$ step $n \geqslant 1$ as

$$
\begin{aligned}
T^{(n)}\left(h, h^{\prime}\right)= & b^{\zeta} \int_{-\infty}^{\infty} \mathrm{d} h_{1} \ldots \int_{-\infty}^{\infty} \mathrm{d} h_{b-1} T^{(n-1)}\left(b^{\zeta} h, h_{1}\right) \\
& \times T^{(n-1)}\left(h_{1}, h_{2}\right) \ldots T^{(n-1)}\left(h_{b-1}, b^{\zeta} h^{\prime}\right),
\end{aligned}
$$

where $b \geqslant 2$ is the rescaling factor and $\zeta$ is the wandering exponent. The normalised interaction is defined as

only a few years ago by Parry et al. [25], who argued that the effective interfacial Hamiltonian for short-range critical wetting in three dimensions is in fact nonlocal, and that in the small gradient limit, $\nabla h \ll 1$, it reduces to that proposed by Fisher et al. [23]. However, and remarkably, a thorough renormalisation-group and computer simulation analysis of the nonlocal Hamiltonian shows no stiffness instability and hence the wetting transition remains continuous [25]. 


$$
\begin{aligned}
& Z\left(h, h^{\prime \prime} ; x\right) \approx Z\left(h, h^{\prime} ; x\right)+\left(h^{\prime \prime}-h^{\prime}\right) \frac{\partial Z\left(h, h^{\prime} ; x\right)}{\partial h^{\prime}}+\frac{\left(h^{\prime \prime}-h^{\prime}\right)^{2}}{2} \frac{\partial^{2} Z\left(h, h^{\prime} ; x\right)}{\partial h^{\prime 2}}, \\
& K\left(h^{\prime \prime}, h^{\prime} ; \Delta x\right) \approx G\left(h^{\prime \prime} ; h^{\prime}, \Delta x\right)\left[1+\Delta x \tilde{v}\left(h^{\prime}, h^{\prime}\right)+\frac{\Lambda^{\prime}\left(h^{\prime}\right)}{4 \Lambda\left(h^{\prime}\right)}\left(h^{\prime \prime}-h^{\prime}\right)-\frac{\Lambda^{\prime}\left(h^{\prime}\right)}{4 \Delta x}\left(h^{\prime \prime}-h^{\prime}\right)^{3}\right. \\
& \left.+\frac{\left(h^{\prime \prime}-h^{\prime}\right)^{2}}{16} \times\left(\frac{\Lambda^{\prime \prime}\left(h^{\prime}\right)}{\Lambda\left(h^{\prime}\right)}-\frac{1}{2}\left(\frac{\Lambda^{\prime}\left(h^{\prime}\right)}{\Lambda\left(h^{\prime}\right)}\right)^{2}\right)-\frac{\left(h^{\prime \prime}-h^{\prime}\right)^{4}}{16 \Delta x}\left(\Lambda^{\prime \prime}\left(h^{\prime}\right)+\frac{\left(\Lambda^{\prime}\left(h^{\prime}\right)\right)^{2}}{\Lambda\left(h^{\prime}\right)}\right)+\frac{\left(h^{\prime \prime}-h^{\prime}\right)^{6}}{32(\Delta x)^{2}}\left(\Lambda^{\prime}\left(h^{\prime}\right)\right)^{2}\right],
\end{aligned}
$$

$\beta H^{(n)}=-\ln T^{(n)}$. Note that this functional renormalisation preserves the symmetry under exchange of the arguments of $H^{(n)}$. This RG scheme is formally exact, but it cannot be solved analytically in general. Instead of solving numerically the $\mathrm{RG}$ recursive equations, we shall analyse the effect of the RG iterations in a subspace of the functional space $\left\{H^{(n)}\right\}$. In particular, we shall consider the transfer matrix to be equal to the propagator corresponding to the continuum limit of the PBD Hamiltonian $H_{e w} \equiv H_{e w}\left[h ; k, \rho, \alpha, a, w_{1}, w_{2}\right]$ given by eq. (4)

$$
T\left(h_{0}, h_{1}\right) \equiv Z\left(h_{0}, h_{1} ; x=1\right)=\int \mathcal{D} h \mathrm{e}^{-\beta H_{e w}},
$$

where we integrate over all the continuum paths $h(t)$ $(0 \leqslant t \leqslant x)$ subject to the conditions $h(0)=h_{0}$ and $h(x)=$ $h_{1}$. Due to the properties of the transfer integral, the application of the RG scheme eq. (7) to this class of Hamiltonians yields

$$
Z^{\prime}\left(h, h^{\prime} ; 1\right)=b^{\zeta} Z\left(b^{\zeta} h, b^{\zeta} h^{\prime} ; b\right),
$$

where $Z^{\prime}\left(h, h^{\prime} ; 1\right)$ is the transfer matrix associated with a new continuum PBD Hamiltonian $H_{e w}^{\prime} \equiv$ $H_{e w}^{\prime}\left[h^{\prime} ; k^{\prime}, \rho^{\prime}, \alpha^{\prime}, a^{\prime}, w_{1}^{\prime}, w_{2}^{\prime}\right]$. The renormalized Hamiltonian parameters are related to the original ones via

$$
\begin{aligned}
& k^{\prime}=k b^{2 \zeta-1}, \quad \rho^{\prime}=\rho, \quad \alpha^{\prime}=b^{\zeta} \alpha, \\
& a^{\prime}=b^{\zeta} a, \quad w_{i}^{\prime}=w_{i} b, \quad i=1,2, \ldots .
\end{aligned}
$$

The wandering exponent is taken as $\zeta=1 / 2$ in analogy to the wetting case, so $k$ and $\rho$ are unchanged by the RG iterations. On the other hand, $a, \alpha,\left|w_{1}\right|$ and $w_{2}$ increase in each RG step. In order to reveal the irrelevance (in the RG sense) of the nonharmonic contribution of the stacking interaction, we shall show that the decimation procedure described above can be related to an analogous RG scheme for a modified PB model (i.e. with $\rho=0$ ). First, we note that the presence of the position-dependent term in the stacking interaction makes the definition of the propagator eq. (8) ambiguous. A similar problem is reported for the quantisation of classical systems with a position dependent mass [31,32]. We choose the following definition of the propagator:

$$
Z\left(h_{0}, h_{b} ; x\right)=\lim _{b \rightarrow \infty} \int \mathrm{d} h_{1} \ldots \mathrm{d} h_{b-1} \prod_{j=1}^{b} K\left(h_{j}, h_{j-1} ; x / b\right),
$$

where $K\left(h, h^{\prime} ; x\right)$ is defined as

$$
\begin{aligned}
& K\left(h, h^{\prime} ; x\right)=\sqrt{\frac{\beta k\left(1+\rho e^{-\alpha\left(h+h^{\prime}\right)}\right)}{2 \pi x}} \\
& \quad \times \exp \left(-\frac{\beta k}{2 x}\left(1+\rho e^{-\alpha\left(h+h^{\prime}\right)}\right)\left(h-h^{\prime}\right)^{2}-x \tilde{v}\left(h, h^{\prime}\right)\right),
\end{aligned}
$$

with a modified potential $\tilde{v}\left(h, h^{\prime}\right)$ given by $[10,11]$

$$
\begin{aligned}
\tilde{v}\left(h, h^{\prime}\right)= & \beta w_{1} e^{-\frac{a}{2}\left(h+h^{\prime}\right)}+\beta w_{2} e^{-a\left(h+h^{\prime}\right)} \\
& +\frac{\beta w_{3}}{2} \ln \left(1+\rho e^{-\alpha\left(h+h^{\prime}\right)}\right)
\end{aligned}
$$

where initially $\beta w_{3}=1$. With this definition the propagator can be understood as the result of a first RG step for large $b$ and $x=b$ before rescaling the distance (see eq. (7)). On the other hand, this expression reduces to the PB case as $\rho \rightarrow 0$. The propagator $Z$ verifies a Schrödingerlike equation. To obtain it, we note that for small $\Delta x$

$$
\begin{aligned}
Z\left(h, h^{\prime} ; x+\Delta x\right) & \approx Z\left(h, h^{\prime} ; x\right)+\Delta x \frac{\partial Z}{\partial x} \\
& \approx \int \mathrm{d} h^{\prime \prime} Z\left(h, h^{\prime \prime} ; x\right) K\left(h^{\prime \prime}, h^{\prime} ; \Delta x\right) .
\end{aligned}
$$

We expand $Z\left(h, h^{\prime \prime} ; x\right)$ and $K\left(h^{\prime \prime}, h^{\prime} ; \Delta x\right)$ for small $\left|h^{\prime \prime}-h^{\prime}\right|$ as

$$
\text { see eqs. (15) and (16) above }
$$

where we defined $\Lambda\left(h^{\prime}\right)=\beta k\left(1+\rho e^{-2 \alpha h^{\prime}}\right), \quad G\left(h^{\prime \prime} ; h^{\prime}\right.$, $\Delta x)=\sqrt{\Lambda\left(h^{\prime}\right) / 2 \pi \Delta x} \exp \left(-\Lambda\left(h^{\prime}\right)\left(h^{\prime \prime}-h^{\prime}\right)^{2} / 2 \Delta x\right)$ and the prime denotes differentiation with respect to the indicated arguments. Due to the Gaussian form of $G\left(h^{\prime \prime} ; h^{\prime}\right.$, $\Delta x)$, we can evaluate trivially the integrals on $h^{\prime \prime}$. In the limit $\Delta x \rightarrow 0$, the resulting expression reduces to

$$
-\frac{\partial Z\left(h, h^{\prime} ; x\right)}{\partial x}=-\frac{1}{2} \frac{\partial}{\partial h^{\prime}}\left(\frac{1}{\Lambda} \frac{\partial Z\left(h, h^{\prime} ; x\right)}{\partial h^{\prime}}\right)+v^{*} Z
$$


with the initial condition $Z\left(h, h^{\prime} ; 0^{+}\right)=\delta\left(h-h^{\prime}\right)$, and where ${ }^{2}$

$$
v^{*}(h)=\tilde{v}(h, h)+\frac{\Lambda^{\prime \prime}(h)}{8 \Lambda^{2}(h)}-\frac{1}{2 \Lambda(h)}\left(\frac{\Lambda^{\prime}(h)}{\Lambda(h)}\right)^{2} .
$$

Introducing the change of variables [33]

$$
\begin{gathered}
\eta=\int \mathrm{d} h \sqrt{\Lambda(h)}, \\
\tilde{Z}\left(\eta, \eta^{\prime} ; x\right)=\Lambda(h)^{-1 / 4} \Lambda\left(h^{\prime}\right)^{-1 / 4} Z\left(h, h^{\prime} ; x\right),
\end{gathered}
$$

eq. (17) yields

$$
-\frac{\partial \tilde{Z}\left(\eta, \eta^{\prime} ; x\right)}{\partial x}=-\frac{1}{2} \frac{\partial^{2} \tilde{Z}\left(\eta, \eta^{\prime} ; x\right)}{\partial \eta^{\prime 2}}+v \tilde{Z}
$$

with the effective potential $v=v(\eta)$ being defined as

$$
\begin{aligned}
v(\eta) & =v^{*}(h(\eta))+\left[\frac{7}{32 \Lambda}\left(\frac{\Lambda^{\prime}}{\Lambda}\right)^{2}-\frac{\Lambda^{\prime \prime}}{8 \Lambda^{2}}\right]_{h=h(\eta)} \\
& =\tilde{v}(h(\eta), h(\eta))-\left.\frac{9}{8 \Lambda}\left(\frac{\Lambda^{\prime}}{\Lambda}\right)^{2}\right|_{h=h(\eta)} .
\end{aligned}
$$

Consequently, the propagator of the PBD model can be mapped onto a propagator of a PB-like model (i.e. $\rho=$ 0 ), where the effect of the position-dependent stacking interaction is absorbed into the definition of the variable $\eta$ and the effective potential $v$. From eq. (19) we obtain the expression for the variable $\eta$

$$
\begin{aligned}
\eta(h ; \alpha, k, \rho)= & \sqrt{\beta k}\left[h+\frac{1}{\alpha}\left(\ln \frac{1+\sqrt{1+\rho e^{-2 \alpha h}}}{2}\right.\right. \\
& \left.\left.-\sqrt{1+\rho e^{-2 \alpha h}}+1\right)\right]
\end{aligned}
$$

so $\eta \sim \sqrt{\beta k} h$ for $h>\alpha^{-1}$. On the other hand, this expression verifies $\eta(\sqrt{b} h ; \alpha, k, \rho)=\sqrt{b} \eta(h ; \sqrt{b} \alpha, k, \rho)$. Finally, the second term in eq. (22) decays exponentially at large distances as

$$
\frac{9}{8 \Lambda}\left(\frac{\Lambda^{\prime}}{\Lambda}\right)^{2}=\frac{9 \alpha^{2} \rho^{2} e^{-4 \alpha h}}{8 \beta k\left(1+\rho e^{-2 \alpha h}\right)^{3}} \sim \frac{9 \alpha^{2} \rho^{2} e^{-4 \alpha h}}{8 \beta k} .
$$

Therefore, the effective potential $v(\eta)$ decays exponentially with $\eta$. Substituting eq. (20) into eq. (9), and taking into account eq. (23), we find that under a RG step the propagator $\tilde{Z}$ renormalizes as

$$
\begin{aligned}
\tilde{Z}^{\prime} & \equiv \tilde{Z}^{\prime}\left(\eta\left(h ; \alpha^{\prime}, k^{\prime}, \rho^{\prime}\right), \eta\left(h^{\prime} ; \alpha, k, \rho\right) ; 1\right) \\
& =\sqrt{b} \tilde{Z}\left(\eta(\sqrt{b} h ; \alpha, k, \rho), \eta\left(\sqrt{b} h^{\prime} ; \alpha, k, \rho\right) ; b\right),
\end{aligned}
$$

\footnotetext{
${ }^{2}$ Note that this Schrödinger-like eq. (18) is not the same as the one proposed in ref. [11], which is in fact associated with a non-Hermitian Hamiltonian operator. Our approach preserves the self-adjointness of the corresponding Hamiltonian operator, as expected from the symmetric character of $K\left(h, h^{\prime} ; \Delta x\right)$.
}

where the renormalized effective potential parameters follow eqs. (10) with $\zeta=1 / 2$. Note that eq. (25) is not the recursion relationship for $2 \mathrm{D}$ RG decimation scheme. However, as we iterate the RG equations, the variable $\eta$ becomes proportional to $h$ as $\alpha^{-1} \rightarrow 0$. Consequently, the high-temperature (HTFP) and critical (CFP) fixed points of the standard decimation RG procedure $T_{\mathrm{HTFP}}^{*}$ and $T_{\mathrm{CFP}}^{*}$, respectively, $[28,34]$

$$
\begin{aligned}
& T_{\mathrm{HTFP}}^{*}=\sqrt{\frac{\beta k}{2 \pi}}\left(e^{-\frac{\beta k\left(h-h^{\prime}\right)^{2}}{2}}-e^{-\frac{\beta k\left(h+h^{\prime}\right)^{2}}{2}}\right), \\
& T_{\mathrm{CFP}}^{*}=\sqrt{\frac{\beta k}{2 \pi}}\left(e^{-\frac{\beta k\left(h-h^{\prime}\right)^{2}}{2}}+e^{-\frac{\beta k\left(h+h^{\prime}\right)^{2}}{2}}\right),
\end{aligned}
$$

are also fixed points for the recursion eq. (25), corresponding to $\alpha^{-1}=0$. Moreover, the RG flow given by eq. (25) differs from the expression for a true decimation only in terms proportional to $\exp (-2 \alpha h)$ for large $\alpha$, which lead to irrelevant corrections in the RG sense. Therefore, the RG flow close to the critical fixed point must be qualitatively similar to that obtained for $\rho=0$, and we conclude that the DNA denaturation transition in the PBD model is continuous and belongs to the 2D short-ranged, critical wetting universality class.

Conclusions. - In this paper we have addressed the question of the order of the DNA denaturation transition for the PBD model. By using an exact decimation procedure, we have shown that the position-dependent stacking interaction is irrelevant in the RG sense, so the transition is continuous and in the same universality class as the $2 \mathrm{D}$ critical wetting for short-ranged forces. However, our analysis only identifies the true asymptotic critical behaviour, not its range. If the universal critical region is narrow enough, numerically obtained denaturation transitions in the PBD model may look like firstorder. For typical values of the parameters, a crossover temperature $T_{\text {cross }} / T_{m} \sim 0.99$ has been numerically estimated, $T_{m}$ being the melting temperature (see fig. 2 of ref. [10]). Thus, the critical region turns out to be very narrow, which is a consequence of the entropic barrier induced by the anharmonicity in the stacking interaction [10]. This would explain the difficulties in determining the order of the transition that have been reported in the literature.

$$
* * *
$$

JMR-E acknowledges financial support from Spanish Ministerio de Ciencia e Innovación and Junta de Andalucía through Grants FIS2009-09326 and P06-FQM01869, respectively. A "Ramón y Cajal" Fellowship from the Spanish Ministerio de Ciencia e Innovación is also gratefully acknowledged. FDLS and MAM acknowledge financial support from Junta de Andalucía through Grants FQM-165 and P09-FQM4682, and from the Spanish Ministerio de Ciencia e Innovación through Grant FIS2009-08451. 


\section{REFERENCES}

[1] Yakushevich L. V., Non-linear Physics of DNA (WileyVCH, Weinheim) 2004, p. 1.

[2] Zimm B. H. and Bragg J. K., J. Chem. Phys., 31 (1959) 526; Wartell R. M. and Benight A. S., Phys. Rep., 126 (1985) 67.

[3] Jost D. and Everaers R., J. Phys.: Condens. Matter, 21 (2009) 034108.

[4] Poland D. and Scheraga H. A., J. Chem. Phys., 45 (1966) 1456.

[5] Peyrard M. and Bishop A. R., Phys. Rev. Lett., 62 (1989) 2755.

[6] Kafri Y., Mukamel D. and Peliti L., Phys. Rev. Lett., 85 (2000) 4988; Eur. Phys. J. B, 27 (2002) 135.

[7] Richard C. and Guttmann A. J., J. Stat. Phys., 115 (2004) 925.

[8] Campa A. and Giansanti A., Phys. Rev. E, 58 (1998) 3585.

[9] Dauxois T., Peyrard M. and Bishop A. R., Phys. Rev. E, 47 (1993) R44. See also, Peyrard M., Nonlinearity 17 (2004) R1.

[10] Cule D. and Hwa T., Phys. Rev. Lett., 79 (1997) 2375.

[11] Theodorakopoulos N., Dauxois T. and Peyrard M., Phys. Rev. Lett., 85 (2000) 6.

[12] Buyukdagli S. and Joyeux M., Phys. Rev. E, 77 (2008) 031903.

[13] Buyukdagli S. and Joyeux M., Phys. Rev. E, 73 (2006) 051910; Joyeux M. and Buyukdagli S., Phys. Rev. E, 72 (2005) 051902; Joyeux M. and Florescu A. M., J. Phys.: Condens. Matter, 21 (2009) 034101.

[14] Zoli M., Phys. Rev. E, 79 (2009) 041927.

[15] Dietrich S., in Phase Transitions and Critical Phenomena, edited by Domb C. and Lebowitz J., Vol. 12 (Academic Press, New York) 1988, p. 1; ScHICK M., Liquids at Interfaces, Les Houches Summer School Proceedings, edited by Charvolin J., JoAnny J.F. and Zinn-Justin J., Vol. 48 (North Holland, Amsterdam) 1990, p. 415.

[16] Fisher M. E., J. Stat. Phys., 34 (1984) 667.
[17] Ares S. and SÁnchez A., Eur. Phys. J. B, 56 (2007) 253.

[18] De los Santos F., Al Hammal O. and Muñoz M. A., Phys. Rev. E, 77 (2008) 032901.

[19] Lipowsky R., J. Phys. A: Math. Gen., 18 (1985) L585.

[20] Brézin E., Halperin B. I. and Leibler J., Phys. Rev. Lett., 50 (1983) 1387; Fisher D. S. and Huse D. A., Phys. Rev. B, 32 (1985) 247.

[21] Binder K., Landau D. P. and Kroll D. M., Phys. Rev. Lett., 56 (1986) 2272; Binder K., LANDAU D. and Müller M., J. Stat. Phys., 110 (2003) 1411.

[22] Ross D., Bonn D. and Meunier J., Nature, 400 (1999) 737.

[23] Fisher M. E. and Jin A. J., Phys. Rev. Lett., 69 (1992) 792. See also Jin A. J. and Fisher M. E. Phys. Rev. B, 48 (1993) 2642.

[24] Boulter C. J., Phys. Rev. Lett., 79 (1997) 1897.

[25] Parry A. O., Romero-Enrique J. M. and Lazarides A., Phys. Rev. Lett., 93 (2004) 086104; PARRY A. O., Romero-Enrique J. M., Bernardino N. R. and RAscón C., J. Physics: Condens. Matter, 20 (2008) 505102.

[26] Nelson D. R. and Fisher M. E., Ann. Phys. (N.Y.), 92 (1975) 226.

[27] Jülicher F., Lipowsky R. and Müller-KrumbhaAR H., Europhys. Lett., 11 (1990) 657.

[28] Huse H. A., Phys. Rev. Lett., 58 (1987) 176.

[29] Spohn H., Europhys. Lett., 14 (1991) 689.

[30] Romero-Enrique J. M. and Parry A. O., J. Phys.: Condens. Matter, 17 (2005) S3487; Europhys. Lett., 72 (2005) 1004; New J. Phys., 9 (2007) 167.

[31] Thomsen J., Einevoll G. T. and Hemmer P. C., Phys. Rev. B, 39 (1989) 12788.

[32] Chetouani L., Dekar L. and Hammann T. F., Phys. Rev. A, 52 (1995) 82.

[33] Yu J. and Dong S.-H., Phys. Lett. A, 325 (2004) 194.

[34] Burkhardt T. W., Phys. Rev. B, 40 (1989) 6987. 